\title{
Construction of a Model and Development of an Algorithm for Solving the Wave Problem under Pulsed Loading
}

\author{
Khabdolda Bolat ${ }^{1}$, Zhuzbayev Serik ${ }^{2}$, Sabitova Diana $S^{3}$ \\ Aitkenova Ailazzat A ${ }^{4}$, Serikbayeva Sandugash ${ }^{5}$, Badekova Karakoz $\mathrm{Zh}^{6}$, Yerzhanova Akbota $\mathrm{Y}^{7}$ \\ Department of Information Systems, L.N. Gumilyov Eurasian National University, Nur-Sultan, Kazakhstan 1, 2, 5 \\ Department of Information Systems and Computer Engineering, Sh.Ualikhanov Kokshetau University ${ }^{3}$ \\ Department of Computer Science and Biostatistics, Karaganda Medical University ${ }^{4,6}$ \\ S. Seifullin Kazakh Agro Technical University, Nur-Sultan, Kazakhstan ${ }^{7}$
}

\begin{abstract}
The article considers approaches and methods for modeling the wave process resulting from blasting operations. The analysis of modeling methods has shown that in the context of the task it is advisable to conduct a study based on the application of the method of behavioral characteristics, which was optimized using the splitting method. The defining equations were calculated, the point scheme of the template was selected, the resolving difference equations for dynamic boundary value problems of a seismic nature were calculated. Based on the method, an algorithm for calculating the relationship between the voltage and the seismic medium was developed, which allowed generating a code and designing an information system for calculating the wave process.
\end{abstract}

Keywords-Information systems; wave process; explosive technologies; method of bicharacteristics; stress tensor

\section{CONDUCTION}

When compacting the foundations of structures, sinking underground developments, the behavior of embankments, dams, dams, etc., explosion energy is widely used. To achieve the maximum effect of blasting, it is necessary to properly study the effect of explosives on soils as a special dynamic effect. In the practice of explosives, both individual charges and very complex systems of them are used, which is undoubtedly of wide technical interest [1,2]. In [3,4], the influence of the pile driving depth on the stress-strain state of the base is studied, the interaction of the pile structure with the ground is studied, and the stresses arising in the piles themselves during driving into the ground are analyzed.

The increasing volume of industrial, mining, hydraulic engineering and aviation engineering makes it necessary to improve the methods of studying wave problems. The known methods of solving problems [5-13] can not always fully reveal the features of contact problems of dynamics.

As part of the study of wave processes using explosive technologies, the problem of propagation of elastic waves under pulsed loading was chosen. To solve this problem, an optimized method of behavioral characteristics is used with the addition of the ideas of the splitting method $[14,15]$. The solution of a number of problems based on this method contributed to the writing of an algorithm and the development of information for the analysis of wave processes in various media [16,17], including using composite materials technology [18].

\section{Problem Statement}

Let a repeated dynamic load (Fig. 2), which is the result of blasting operations, act on the resting quarter plane with the insert (Fig. 1) at some depth from the free surface and at some distance from the insert. At the same time, a shock wave propagates from the source of the explosion, which, as it moves away from it, turns into a continuous compression wave.

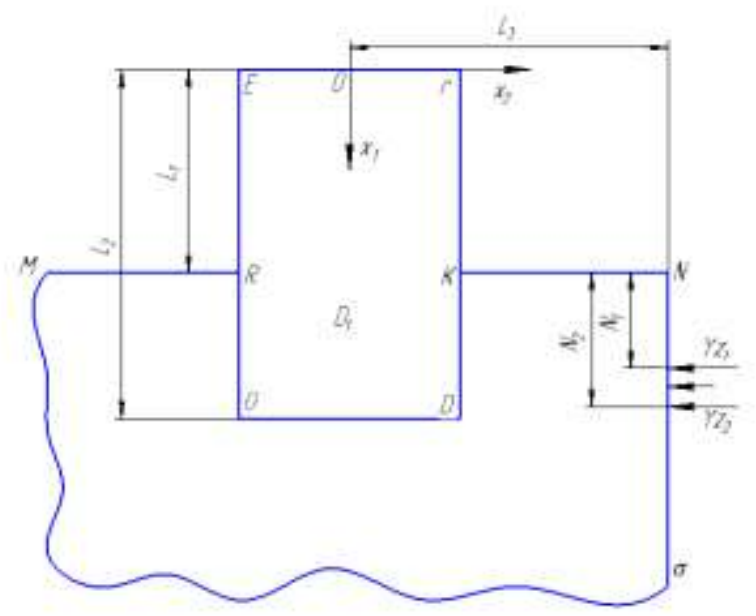

Fig. 1. The Study Area.

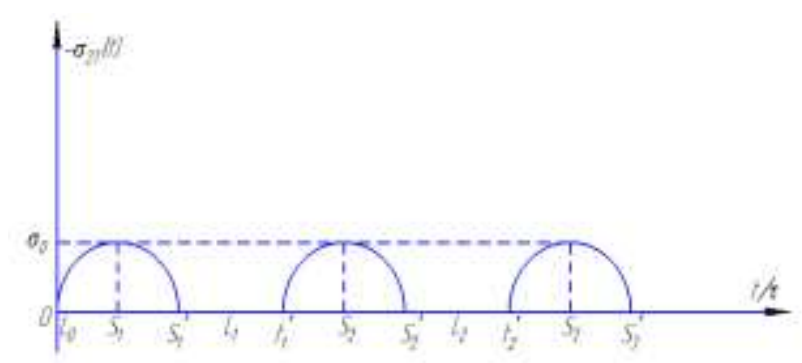

Fig. 2. The Shape of the Current Load. 
It reaches the insertion and determines its tense state. There are many technical problems here, for example, insertion fluctuations. In any case, the initial urgent task is to study the features and critical values of stress fields in the perturbed region [24].

In mathematical modeling of the problem of underground blasting, the soil is considered as an elastic medium occupying a quarter of the plane, and the insert as an elastic structure. The shock load is represented as a harmonic distributed load that acts along the normal to the vertical surface of the quarter plane on a certain section. In the Cartesian coordinate system $x_{i}(i=1,2)$ the rectangular insert (body 1) occupies the area $D_{1}\left(0 \leq x_{1} \leq L_{2} \cap\left|x_{2}\right| \geq l\right)$ and the quarter plane (body $2)$ is the area $D_{2}\left(L_{1} \leq x_{1} \leq \infty \cap-\infty \leq x_{2} \leq L_{3}\right)$ (Fig. 1), and the quarter plane (body 2$)$ is the $D_{2}\left(L_{1} \leq L_{2} ; l \geq L_{3}\right)$.

The boundary value problem assumes that the initial:

$v_{\alpha}^{(k)}=\sigma_{\alpha j}^{(k)}=0,(\alpha, j=1,2 ; k=1,2)$,

boundary conditions:

$\sigma_{1 j}^{(2)}=0,(j=1,2)$ by $x_{1}=L_{1}, l \leq\left|x_{2}\right|<\infty$

$\sigma_{j 2}^{(1)}=0,(j=1,2)$ by $0 \leq x_{1} \leq L_{1},\left|x_{2}\right|=l$

and contact conditions:

$v_{\alpha}^{(1)}=v_{\alpha}^{(2)}, \sigma_{1 j}^{(1)}=\sigma_{1 j}^{(2)},(\alpha, j=1,2)$ by $x_{1}=L_{2},\left|x_{2}\right| \leq l$

$v_{\alpha}^{(1)}=v_{\alpha}^{(2)}, \sigma_{j 2}^{(1)}=\sigma_{j 2}^{(2)},(\alpha, j=1,2)$ by $L_{1} \leq x_{1} \leq L_{2},\left|x_{2}\right|=$ $l$

At the boundary $E F\left(x_{1}=0,\left|x_{2}\right| \leq 1\right)$, the stress-free surface conditions are assumed (Fig. 1).

$\sigma_{11}^{1}=0, \sigma_{12}^{1}=0$

It is assumed that the NB boundary $N B\left(0 \leq x_{1} \leq\right.$ $\infty, x_{2}=L_{3}$ ) is also free from stresses (3) and only on the local section $\left(N_{1} \leq x_{1} \leq N_{2}, x_{2}=L_{3}\right)$ of the NB boundary of the quarter plane, the normal component $\sigma_{22}^{2}$ acts at certain time intervals, changing according to the la.

$\sigma_{22}^{2}(t)=\left\{\begin{array}{c}0, t \leq 0 S_{i} \leq t \leq t_{i}^{*} \\ A_{0} \sin \left(\frac{\omega\left(t-S_{i}\right)}{B_{0}}\right), t_{i-1}^{*} \leq t \leq S_{i}\end{array}\right.$

$\sigma_{12}^{2}(t)=0$,

where $\quad i=1,2, \ldots, n ; B_{0}=S_{i}-t_{i-1}^{*} ; S_{i}=\frac{B_{0}}{2+t_{i-1}^{*}} . \quad$ The amplitude $A_{0}$ and the frequency $\omega$ the oscillation frequency remains constant. The boundary conditions (7) simulate the conditions of blasting operations in the well, acting at a certain $\left(x_{1} \leq L_{1}, x_{2}=L_{3}-l\right.$ ) distance from the structure $D_{1}$.

Numerical calculations of the problem were carried out with the following initial data: $f(t)=-A_{0} t \exp (-t)$ by $t \geq$ $0 ; \tau=\Delta t=0.002 ; l=10 h ; L_{1}=10 h ; L_{2}=20 h ; h=\Delta x_{1}=$ $\Delta x_{2}=0.05$. External loads $A_{0}=-0.5, \omega=9$. The insertion configuration (region $\mathrm{D}_{1}$ )) and the local impact site varied.
Under the described conditions, it is necessary to investigate the stress-strain state of an inhomogeneous medium $D_{1} \cap D_{2}$.

\section{METHOD OF BeHAVIORAL CHARACTERISTICS WITH THE IDEAS OF THE SPLITTING METHOD}

To solve this problem, along with the initial (1) and boundary conditions (1) - (5), a system of equations consisting of equations of motion and relations of the generalized Hooke's law is used.

$\rho_{k} \ddot{u}_{\alpha}^{(k)}=\sigma_{\alpha \beta, \beta}^{(k)}$

$\sigma_{\alpha j}^{(k)}=\lambda_{k} \varepsilon_{\beta \beta}^{(k)} \delta_{\alpha j}+2 \mu_{k} \varepsilon_{\alpha j}^{(k)}$

where $\varepsilon_{\alpha j}^{(k)}=0.5\left(u_{\alpha, j}^{(k)}+u_{j, \alpha}^{(k)}\right) ; \delta_{\alpha}-$ Kronecker symbol, $u_{\alpha}^{(k)}, \varepsilon_{\alpha j}^{(k)}$ - components of the displacement vector and the strain tensor.

It is convenient to calculate the solution of the problem in a dimensionless space of variables and the desired parameters, which are obtained after the introduction of the notation described in [19].

\section{A. Defining Equations of the Dynamic Problem of the Theory of Elasticity}

Using the relations from [19] for dimensionless quantities, one can obtain $(\mathrm{i} \neq \mathrm{j})$ from equations (8) after simple transformations:

$\rho_{k} \dot{v}_{\alpha}^{(k)}=\sigma_{\alpha \beta, \beta}^{(k)} ; \dot{\sigma}_{\alpha j}^{(k)}=\left(\gamma_{11}^{(k)} v_{j, j}^{(k)}+\gamma_{33}^{(k)} v_{i, i}^{(k)}\right) \delta_{\alpha j}+$ $\gamma_{12}^{(k)}\left(v_{\alpha, j}^{(k)}+v_{j, \alpha}^{(k)}\right)\left(1-\delta_{\alpha j}\right)$

Equations (9) are a linear homogeneous hyperbolic system of first-order differential equations with constant coefficients [16]. Its characteristic surfaces in three-dimensional space $\left(x_{1}, x_{2} ; t\right)$ are hyperconuses with axes parallel to the time axis (Fig. 3 ).

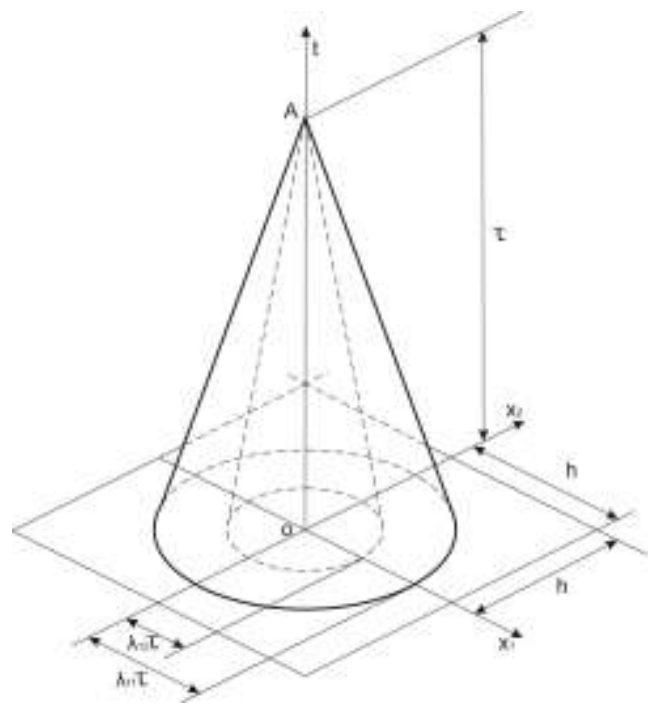

Fig. 3. Characteristic Cones on the Plane. 
The system of equations (9) has two families of characteristic cones. These cones coincide with the behavioral characteristics of equations (9). Along the behavioral characteristics lying in the plane $x_{i}=$ const, equations (9) are functions of only two variables $\left(x_{j} ; t\right)$. This circumstance indicates that the conditions on the behavioral characteristics can be obtained as conditions on the characteristics in the corresponding one-dimensional problem. The corresponding transformations can be performed if one of the spatial variables is alternately fixed in the system of equations (9) [20]. In this case, the system of equations (9) is split into two systems of equations corresponding to the directions $j=1$ and $\mathrm{j}=2(\mathrm{i} \neq \mathrm{j})$ :

$\dot{v}_{\alpha}^{(k)}-\rho_{k}^{-1} \sigma_{\alpha j, j}^{(k)}=A_{\alpha i}^{(k)}$

$\dot{\sigma}_{\alpha j}^{(k)}-\gamma_{\alpha j}^{(k)} v_{\alpha j}^{(k)}=B_{\alpha i}^{(k)}$

where the notation is entered:

$A_{\alpha i}^{(k)}=\rho_{k}^{-1}\left(\sigma_{\alpha \beta, \beta}^{(k)}-\sigma_{\alpha j, j}^{(k)}\right)$

$B_{\alpha i}^{(k)}=\gamma_{33}^{(k)}\left(v_{\alpha, \alpha}^{(k)}-v_{\alpha, j}^{(k)}\right) \delta_{\alpha j}-\gamma_{12}^{(k)}\left(v_{i, j}^{(k)}-v_{j, i}^{(k)}-v_{\alpha, j}^{(k)}\right)(1-$

$\left.\delta_{\alpha j}\right)$

The differential equations of characteristics have the form:

$d x_{\alpha}= \pm \lambda_{\alpha j}^{(k)} d t$

and the conditions on the bicharacteristics are:

$d \sigma_{\alpha j, j}^{(k)} \pm(-1) \rho_{k} \lambda_{\alpha j}^{(k)} d v_{\alpha}^{(k)}=\left(B_{\alpha i}^{(k)} \pm(-1) \rho_{k} \lambda_{\alpha j}^{(k)} A_{\alpha i}^{(k)}\right) d t$

There $\lambda_{\alpha j}^{(k)}=\gamma_{11}^{(k)}$ if $\alpha=\mathrm{j}$ and $\lambda_{\alpha j}^{(k)}=\gamma_{12}^{(k)}$ if $\alpha \neq \mathrm{j}$. It can be seen from (12) that on each of the two hyperplanes there are two pairs of families of bicharacteristics that determine the longitudinal $\lambda_{i i}^{(k)}$ and $\operatorname{shear} \lambda_{i j}^{(k)} \quad(i \neq j ; i, j=1,2)$ wave propagation velocities (Fig. 3). In each of the two planes $\left(\mathrm{x}_{\mathrm{i}} ; \mathrm{t}\right)$ there are two families of behavioral characteristics of the positive and negative directions. The upper sign corresponds to the behavioral characteristics of the positive, and the lower sign corresponds to the negative directions. Equations (12) and (13) correspond to each other with the same pair of indices and with the same arrangement of signs. Equations (10) and conditions (13) are used to find a solution to the formulated problem (1) -(5) [23].

\section{B. Selecting the Point Scheme of the Template}

To perform numerical calculations of the formulated problem for a region with a given configuration $D_{1} \cap D_{2}$, it is necessary to study the characteristic surfaces. The body $D_{1} \cap$ $D_{2}$ is exposed to non-stationary loads. The initial conditions (1) are given by stresses and displacement velocities in the entire body, and the boundary conditions are given by stresses on the surface (2) - (3). It is assumed that both are continuous differentiable functions. The shape of the body is such that it admits the existence of a coordinate system $x_{i}(i=1,2)$, in which the boundary surfaces are coordinate [25].

Let the body $D_{1} \cap D_{2}$ be divided into cells formed by intersections of coordinate $\operatorname{surfaces} x_{i}=\operatorname{const}(i=1,2)$. The linear dimensions of these cells in the direction of the axes $x_{1}$ and $x_{2}$ are considered uniform and equal to $h$. The intersections of the lines $x_{i}=$ const $(i=1,2)$ form nodes. At these nodal points, the values of the desired functions $v_{\alpha}^{(k)}, \sigma_{\alpha j}^{(k)}(\alpha, j=1,2)$ are found at various time points $t_{n}-\tau, t_{n}, t_{n}+\tau(n=1,2, \ldots, N)$ in time increments $\tau$.

A template consisting of a node $O$ and points $E_{\alpha j}^{ \pm(k)}$ lying on the coordinate lines $x_{j}=$ const and spaced from the point $O$ at distances $\lambda_{11}^{(k)} \tau$ and $\lambda_{12}^{(k)} \tau$ is accepted (Fig. 4). Oblique lines originating from point $\mathrm{A}$ are bicharacteristics. In the future, the values of the functions at point $O$ are assigned the upper sign "0"; at points $E_{\alpha j}^{ \pm(k)}$ - the lower and upper signs \pm (for example, $\sigma_{\alpha j}^{ \pm(k)}$ ), and at point $\mathrm{A}$ an additional index is not assigned [21-24].

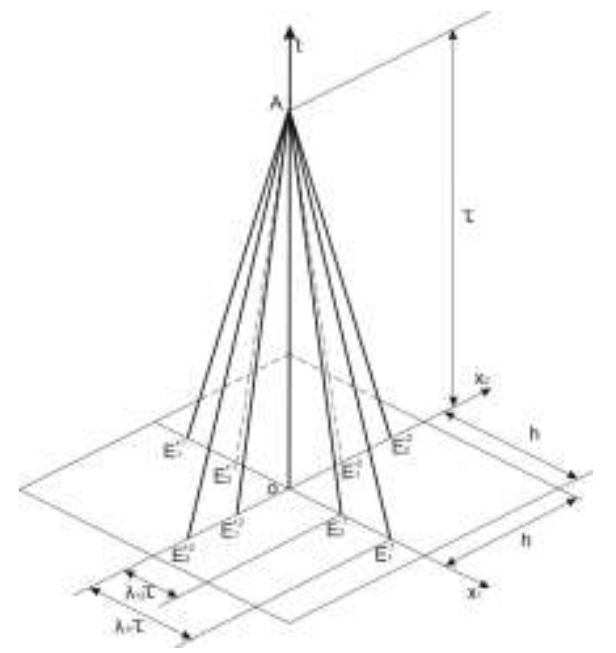

Fig. 4. Point Grid of the difference Scheme for Internal Points.

Based on the described point schemes, the method developed below for solving dynamic problems allows us to determine the particle velocities $v_{\alpha}^{(k)}$ and the components of the stress tensor $\sigma_{\alpha j}^{(k)}$ at point $A$ on the calculated layer in time $t_{n}$ if their values are known on the previous layert $t_{n-1}(n=$ $1,2, \ldots, N)$ at point $\mathrm{O}$ and at adjacent points $E_{\alpha j}^{ \pm(k)}$. Difference schemes of this type are called explicit. Explicit schemes are convenient because there are no difficulties in solving the systems of difference equations associated with them. These systems are solved sequentially from one-time layer to the next. In this case, the desired values at each node point, unlike the implicit difference scheme, are calculated independently of the others [20].

\section{Resolving difference Equations for Solving Dynamic Boundary Value Problems}

Resolving difference equations at internal points. The calculation algorithm of the second order of accuracy is constructed below [20]. The integration of the system of equations (10) from point $\mathrm{O}$ to point $\mathrm{A}$ and the relations (13) from point $E_{\alpha j}^{ \pm(k)}$ to point A by the trapezoid method (Fig. 4) allows us to obtain expressions of the following type: 
$v_{\alpha}^{(k)}=v_{\alpha}^{0(k)}+\frac{\tau}{2}\left(\dot{v}_{\alpha}^{0(k)}+\rho_{k}^{-1} \sigma_{\alpha j, j}^{(k)}+A_{\alpha i}^{(k)}\right) ;$

$\sigma_{\alpha j}^{(k)}=\sigma_{\alpha j}^{0(k)}+\frac{\tau}{2}\left(\dot{\sigma}_{\alpha j}^{0(k)}+\gamma_{\alpha j}^{(k)} v_{\alpha j}^{(k)}+B_{\alpha i}^{(k)}\right)$

and

$\sigma_{\alpha j, j}^{(k)}-\sigma_{\alpha j}^{ \pm(k)} \pm(-1) \rho_{k} \lambda_{\alpha j}^{(k)}\left(v_{\alpha}^{(k)}-v_{\alpha}^{ \pm(k)}\right)=$

$=\frac{\tau}{2}\left(B_{\alpha i}^{(k)}+B_{\alpha i}^{ \pm(k)} \pm(-1) \rho_{k} \lambda_{\alpha j}^{(k)}\left(A_{\alpha i}^{(k)}+A_{\alpha i}^{ \pm(k)}\right)\right.$

where the unknown values at point $A$ are taken without additional indexes.

The values of functions at non-node points $E_{\alpha j}^{ \pm(k)}$ are replaced by the values calculated by the Taylor formula with first-order accuracy for the functions $A_{\alpha i}^{ \pm(k)}$ and $B_{\alpha i}^{(k)}$ and with second-order accuracy for the functions $v_{\alpha}^{(k)}$ and $\sigma_{\alpha j}^{(k)}$ through their values at the node points $O\left(x_{1}, x_{2} ; t\right)[20]$ :

$A_{\alpha i}^{ \pm(k)}=A_{\alpha i}^{0(k)} \pm(-1) \lambda_{\alpha j}^{(k)} \tau \frac{\partial A_{\alpha i}^{(k)}}{\partial x_{j}}$

$B_{\alpha i}^{ \pm(k)}=B_{\alpha i}^{0(k)} \pm(-1) \lambda_{\alpha j}^{(k)} \tau \frac{\partial B_{\alpha i}^{(k)}}{\partial x_{j}}$

and

$\sigma_{\alpha j}^{ \pm(k)}=\sigma_{\alpha j}^{0(k)} \pm(-1) \lambda_{\alpha j}^{(k)} \tau \frac{\partial \sigma_{\alpha j}^{0(k)}}{\partial x_{j}}+\frac{1}{2}\left(\lambda_{\alpha j}^{(k)} \tau\right)^{2} \frac{\partial^{2} \sigma_{\alpha j}^{0(k)}}{\partial x_{j}^{2}}$

$v_{\alpha}^{ \pm(k)}=v_{\alpha}^{0(k)} \pm(-1) \lambda_{\alpha j}^{(k)} \tau \frac{\partial v_{\alpha}^{0(k)}}{\partial x_{j}}+\frac{1}{2}\left(\lambda_{\alpha j}^{(k)} \tau\right)^{2} \frac{\partial^{2} v_{\alpha}^{0(k)}}{\partial x_{j}^{2}}$

Partial derivatives of the system of equations (10) with respect to the variable $\mathrm{x}_{\mathrm{j}}$ are written as:

$\frac{\partial \dot{v}_{\alpha}^{0(k)}}{\partial x_{j}}=\rho_{k}^{-1} \frac{\partial \sigma_{\alpha j, j}^{0(k)}}{\partial x_{j}}+\frac{\partial A_{\alpha i}^{0(k)}}{\partial x_{j}}$

$\frac{\partial \dot{\sigma}_{\alpha j}^{0(k)}}{\partial x_{j}}=\gamma_{\alpha j}^{(k)} \frac{\partial v_{\alpha j}^{(k)}}{\partial x_{j}}+\frac{\partial B_{\alpha i}^{(k)}}{\partial x_{j}}$

Substituting the relations (16), (17) in (15), then excluding with the help of (14) the variables $v_{\alpha}^{(k)}, \sigma_{\alpha j}^{(k)}$ and taking into account (18), we can obtain eight equations with respect to the derivatives $v_{\alpha j}^{(k)}, \sigma_{\alpha j, j}^{(k)}, A_{\alpha i}^{(k)}, B_{\alpha i}^{(k)}$ :

$\gamma_{\alpha j}^{(k) 2} v_{\alpha j}^{(k)} \pm(-1) \lambda_{\alpha j}^{(k)} \sigma_{\alpha j, j}^{(k)}=$

$=\gamma_{\alpha j}^{(k) 2}\left(v_{\alpha j}^{0(k)}+\tau \frac{\partial \dot{v}_{\alpha}^{0(k)}}{\partial x_{j}}\right) \pm \lambda_{\alpha j}^{(k)}\left(\sigma_{\alpha j, j}^{0(k)}+\tau \frac{\partial \dot{\sigma}_{\alpha j}^{0(k)}}{\partial x_{j}}\right)$

Adding and subtracting the corresponding pairs of equations (19) in turn, we can find unknown derivatives:

$v_{\alpha j}^{(k)}=v_{\alpha j}^{0(k)}+\tau \frac{\partial \dot{v}_{\alpha}^{0(k)}}{\partial x_{j}} ;$
$\sigma_{\alpha j, j}^{(k)}=\sigma_{\alpha j, j}^{0(k)}+\tau \frac{\partial \dot{\sigma}_{\alpha j}^{0(k)}}{\partial x_{j}}$
The system of equations (20) can be used to determine unknown derivatives, both in the internal and boundary nodal points of the studied region $D_{1} \cap D_{2}$. Such expressions can be obtained directly by integrating the system of equations (9) according to the Euler scheme, having previously differentiated them by $\mathrm{x}_{\mathrm{i}}$. However, it is important to have intermediate relations (19), which are used in solving systems of equations where boundary functions are given. The substitution of equalities (20) in (14) allows us to obtain unknown functions $v_{\alpha}^{(k)}, \sigma_{\alpha j}^{(k)}$ at the internal nodal points of an inhomogeneous body at time $t_{n-1}+\tau(n=1,2, \ldots, N)[21-$ 22].

\section{ANALYSIS OF THE RESUlT}

Based on the developed information system, the calculation results were obtained, shown in Fig. 5 to 7 , when $l=5 h, L_{1}=5 h, L_{2}=10 h, N_{1}=8 h, N_{2}=11 h$, calculation results.

Fig. 5 shows the changes in the normal stresses along $\sigma_{22}^{k}(k=1,2)$ the $\frac{x_{2}}{h}$ coordinate at time $\mathrm{t}=60 \tau$ in different sections:

$1-x_{1}=5 h$ (this section passes through both the insert and the quarter plane);

$2-x_{2}=10 h$ (the cross-section runs along a quarter of the plane);

$2^{\prime}-x_{1}=10 h$ (the cross-section passes through the insert);

$3-x_{1}=0 h$ (the cross-section passes through the insert).

The normal stresses $\sigma_{22}^{k}(k=1,2)$ in Section 1 near the angular point $N\left(x_{1}=L_{1}, x_{2}=L_{3}\right)$, at the boundary of the free surface $P N\left(x_{1}=L_{1}, l \leq x_{2} \leq L_{3}\right.$ and in the insertion region $\left(x_{1}=L_{1},\left|x_{2}\right| \leq l\right)$ change abruptly with different local extrema. The normal stresses $\sigma_{22}^{2}$ in the section 2 in the quarter plane reach the maximum extreme value $(-0.22)$. It is caused by a given load. On the contact surface of the insert and the quarter plane, the normal stresses $\sigma_{22}^{k}(k=1,2)$ suffer a break, taking different values at the insertion points and at the points of the quarter plane. At the exit from the contact area, the normal voltages $\sigma_{22}^{2}$ again take on a local extreme value. It should be noted that in the contact region, the value of the extremum of the normal voltage $\sigma_{22}^{1}$ (in the Section 2') is almost twice as high as its value in the material of the quarter plane. This result is associated with a greater rigidity of the insert material.

The evolutions of the wave pattern of tangential stresses $\sigma_{12}^{k}(k=1,2)$ at times $\mathrm{t}=40 \tau$ and $\mathrm{t}=60 \tau$ are shown in Fig. 6 and 7.

The isolines constructed in the figures are in good agreement with the nature of the change in pulse loads specified at the boundary with a quarter of the plane. At time $\mathrm{t}=40 \tau$ (Fig. 6), the leading edge of the boundary wave reaches the middle of the insert ( $\mathrm{x}_{1}=0$ axis), i.e., the wave has passed the distance $\mathrm{x}_{2}=20 \mathrm{~h}$ with some delay due to the weakening of elastic perturbations during the transition to the insert material. This moment of time $\mathrm{t}=40 \tau$ corresponds to the action 
time of the first pulse of the boundary load. The isolated traces of extensive single extremums in the entire region $D_{1} \cap D_{2}$ of an inhomogeneous medium are explained by the influence of angular points, points of discontinuity of boundary conditions and contact surfaces.

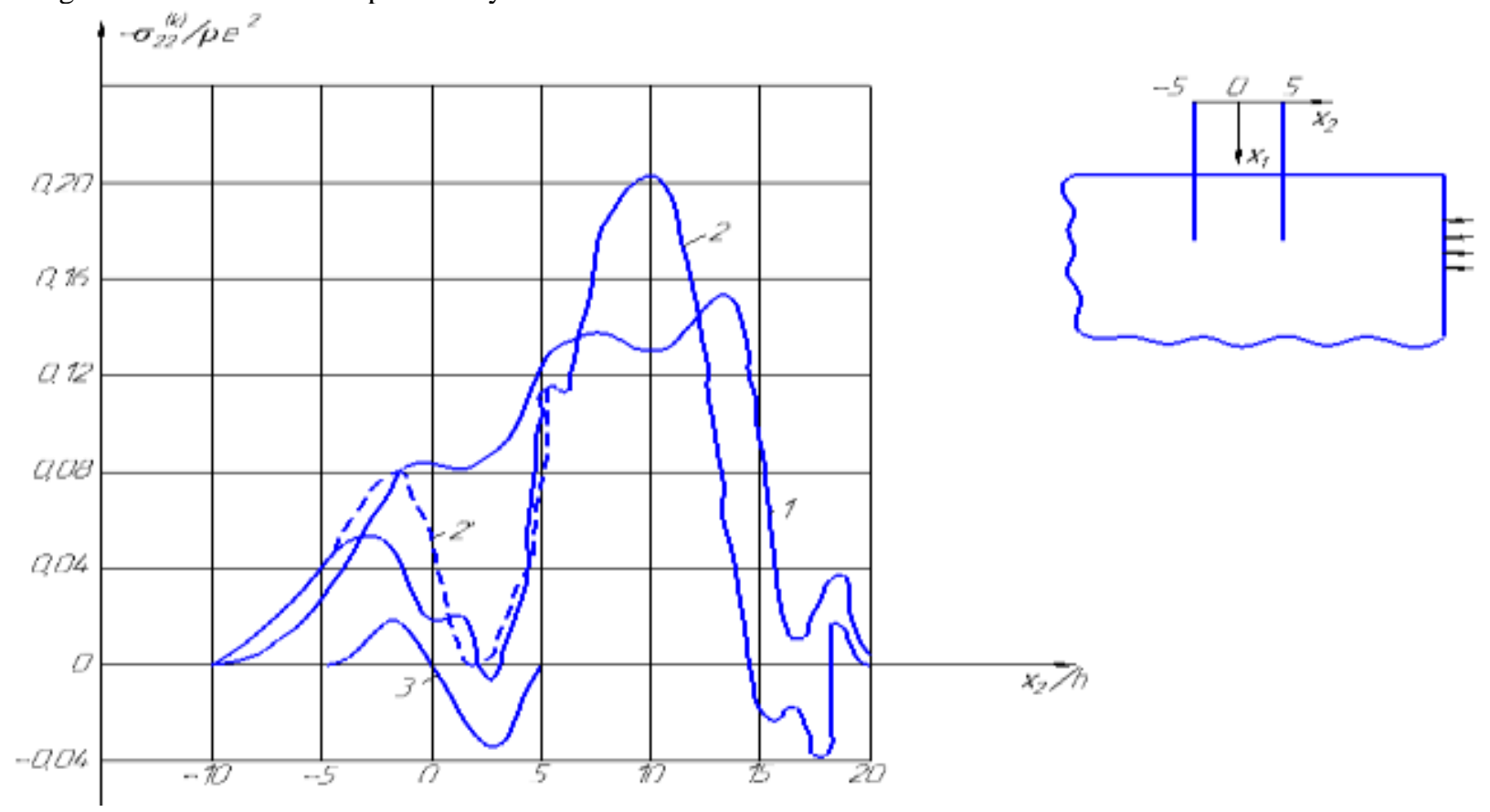

Fig. 5. Change of Normal Stresses $\sigma_{22}^{k}$ at Time $\mathrm{t}=60 \tau$ along the $x_{2}$ Coordinate in Sections $1-x_{1}=5 h, 2-x_{2}=10 h, 2^{\prime}-x_{1}=10 h, 3-x_{1}=0 h$.

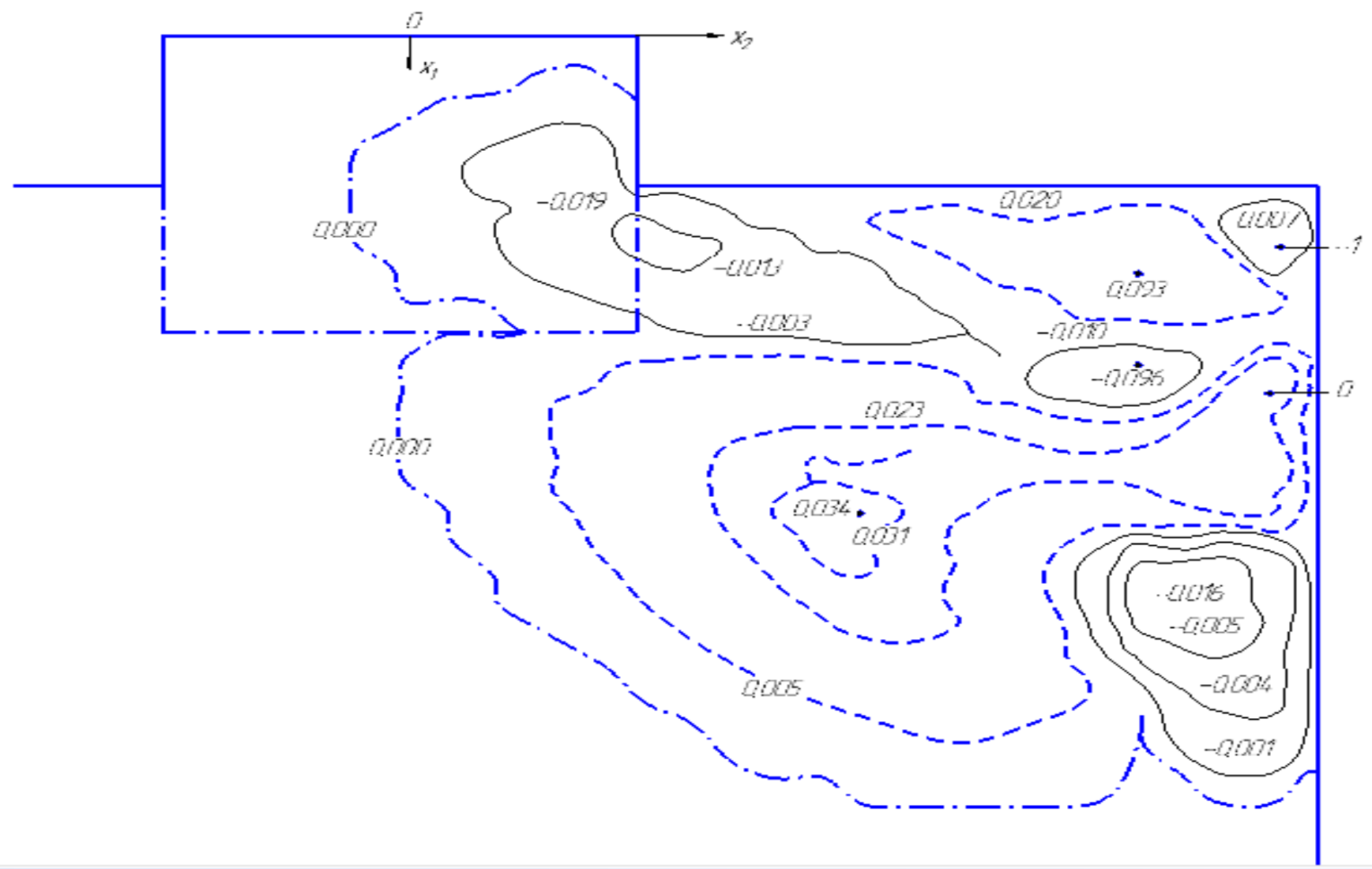

Fig. 6. Tangential Stress Isolines $\sigma_{12}^{k}(k=1,2)$ at Time $\mathrm{t}=40 \tau$. 


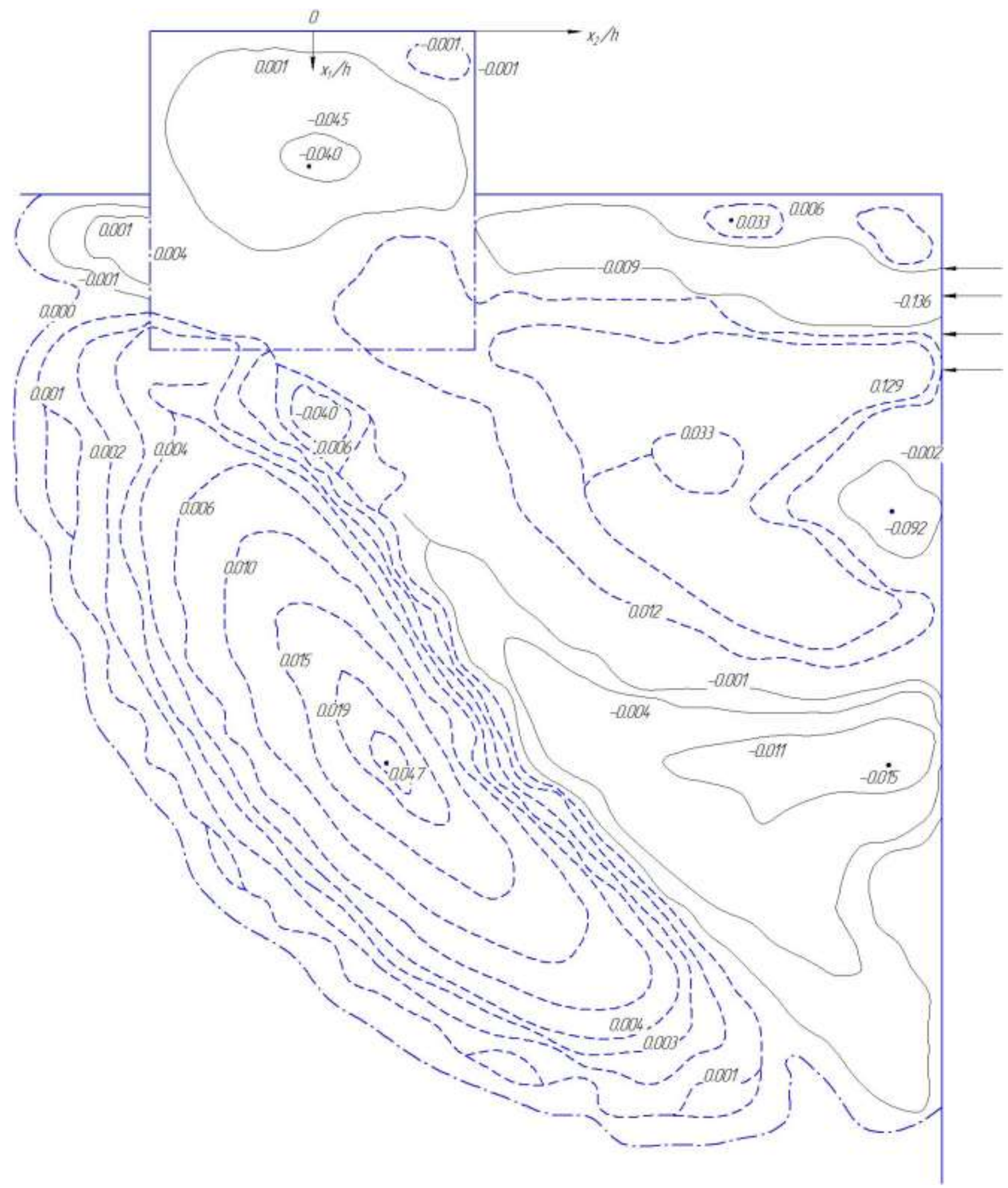

Fig. 7. Tangential Stress Isolines $\sigma_{12}^{k}(k=1,2)$ at Time $\mathrm{t}=60 \tau$.

In Fig. 7, the tangential stresses $\sigma_{12}^{k}(k=1,2)$ reach their maximum values in absolute magnitude near the load application points $\left(N_{1} \leq x_{1} \leq N_{2}, x_{2}=L_{3} ;\right.$; line $\left.B_{1} B_{2}\right)$. At this moment of time $\mathrm{t}=60 \tau$, the second pulse is affected. This can explain the appearance of the selected double zones of stress extremes in the entire region of the inhomogeneous medium $D_{1} \cap D_{2}$. 


\section{CONCLUSION}

The developed information system made it possible to visualize the results of calculating wave propagation during blasting operations, to obtain graphs of changes in normal stresses and tangential stress isoclines [26].

Scientific and theoretical interest in the study of the relationship between stress (stress tensor) and deformations occurring in the medium as a result of blasting remains very relevant. The problems of seismically unstable areas require consideration of this issue from the point of view of modeling the wave process.

Modeling the reaction of the soil to changes resulting from an explosion, using information and communication technologies, allows solving problems not only of an economic, construction, geological nature, but also of the safety of human life.

\section{REFERENCES}

[1] Semenova V. Y. modeling of the response of soil under seismic mikroraionirovanii construction sites // Geophysical journal. — 2015. V. 37, № 1. - P. 137-153.

[2] Juzbaev S. S., Bayaliev B. T., Mortalin N. To. the calculation of the strain state of the system "structure-soil" under the influence of energy strombolian explosions // abstracts of the Republican scientific-practical conference on problems of construction on the landing grounds in southern Kazakhstan. Shymkent, 1991, p. 60-68.

[3] Sabitova D.S., Zhuzbayev S.S., Juzbayeva B.G. Development of an information analysis system for analyzing wave processes in a homogeneous medium // International Journal of Mechanical Engineering and Technology. - 2018. -Vol. 9(12). - P. 499-508.

[4] Akhmetova Z., Zhuzbaev S., Boranbayev S., Sarsenov B. Development of the System with Component for the Numerical Calculation and Visualization of Non-Stationary Waves Propagation in Solids. IOS Book Series: Frontiers in Artificial Intelligence and Applications (FAIA), 2016, Volume293, pp. 353-359, ISSN 0922-6389.

[5] Clifton R. Difference method in plane problems of dynamic elasticity // Mechanics. Collection of translations. 1968. - No. 1. - pp. 103-122.

[6] Tarabrin G. T. Difference schemes of wave problems of elasticity theory: monograph / G. T. Tarabrin; VolgSTU. - Volgograd: RPK "Polytechnic", 2000. - 148 p.

[7] Kukudzhanov V.N. Numerical Continuum Mechanics. De Gruyter, 2012. XVIII, 425 pages.

[8] Reker V. V. Applied mechanics// Series E.-1970. - No. 1.-B. 121-129.

[9] Italien S. M., Masanov, J. K., Baimahanov I. B., N. Makhmetova.M.//Numerical methods for solving problems in solid mechanics, Karaganda, 1987. pp. 3-15.

[10] Bazhenov V. G., gonik E. G., Kibar A. I., D. V. Shoshin Stability and limit state elastic-plastic spherical shells under static and dynamic loadings. Applied Mechanics and Technical Physics, 2014. T. 55, No. 1, pp. 13-22.

[11] Aitaliev Sh. M., Alekseeva L. A., Dildabaev Sh. A., Zhanbyrbaev N. B. The method of boundary integral equations in problems of dynamics of elastic multi-connected bodies. - Alma-Ata: Gylym, 1992. - p. 228.
[12] S. Tleukenov, A. Bobeev, D. Sabitova. Structure of the matriciant for systems of ordinary differential equations of first order and its applications. International journal of applied mathematics \& statistics. 2018. - V.57 (1). - P. 209-217.

[13] Rysbaiuly B., Yunicheva N., Rysbayeva N. An iterative method to calculate the thermal characteristics of the rock mass with inaccurate initial data. Open Engineering, 2016, 6(1).

[14] Zhuzbaev S. S., Sabitova D. S. Computer modeling of elastic wave propagation in a homogeneous medium. BULLETIN OF THE PSU. Physics and Mathematics Series, 2018, No. 1, pp. 68-82. ISSN: 18111807.

[15] Akhmetova Z., Zhuzbaev S., Boranbayev S. The method and software for the solution of dynamic waves propagation problem in elastic medium. Acta Physica Polonica A, Polish Academy of Sciences. -2016, Vol.130. - pp. 352-354, ISSN 0587-4246.

[16] Zhuzbayev S., Sabitova D., Sarsenov B. Computer mathematical modeling of wave processes // 4th intern. conf. on computer and technology applications (ICCTA). - Istanbul, 2018. - P. 1-5.

[17] Zhuzbayev S., Sabitova D. Mathematical modeling of elastic wave propagation in a homogeneous media // Вестник ВосточноКазахстанского государственного технического университета им. Д. Серикбаева. - 2018. - Т. 1, №3, ч. 3. - С. 78-90.

[18] Zhuzbayev S., Adilova A., Akhmetzhanova S., Juzbayeva B., Sabitova D. Design of composite materials using information technology. Journal of Theoretical and Applied Information Technology, 2020, 98(18), c. 3698-3711.

[19] Ashirbayev N. K. Features of the propagation of dynamic perturbations in bodies with inhomogeneities. / / Dissertation work for the degree of Candidate of Physical and Mathematical Sciences, Shymkent, 1986, 207 p.

[20] Tarabrin G. T. Numerical solution of nonstationary problems of dynamics of an anisotropic elastic medium. / / Moscow, Izv. of the USSR Academy of Sciences, Mechanics of a solid body, 1982, No. 2, pp. 83-95.

[21] Y. Ru, G.F. Wang, T.J. Wang, "Diffractions of elastic waves and stress concentration near a cylindrical nano-inclusion incorporating surface effect," Journal of Vibration and Acoustics, vol. 131, 2009: Art. 061011.

[22] J.-Y. Kim, "On the generalized self-consistent model for elastic wave propagation in composite materials," International Journal of Solids and Structures, 2004, pp. 4349-4360.

[23] L. Ya. Cosachevskiy, "On propagation of elastic waves in twocomponent media”, Applied Mathematics and Mechanics, vol. 23, 1959, pp. 1115-1123.

[24] S.K.Serikbayeva, D.A.Tussupov, M.A.Sambetbayeva, A.S. Yerimbetova, Taszhurekova ZH.K., Borankulova G.S. EduDIS construction technology based on Z39.50 protocol: Journal of Theoretical and Applied Information Technology, 31st May 2021 г.. - T. - Vol. 99. No. 10, P.2224-2255.

[25] Serikbayeva S.K, Batyrhanov A.G., Sambetbayeva M.A., Sadirmekova Zh.B., Yerimbetova A.S. Development of technology to support large information storage and organization of reduced user access to this information: (IJACSA) International Journal of Advanced Computer Science and Applications, 2021 г.. - 7 : T. 12. - стр. 493-503.

[26] S.K. Kanaun, V.M. Levin, F.J. Sabina, "Propagation of elastic waves in composites with random set of spherical inclusions (effective medium approach)," Wave Motion, vol 40(1), 2004, pp. 69-88. 